K. TOHGE

KODAI MATH. J.

13 (1990), 101-120

\title{
ON A PROBLEM OF HINKKANEN ABOUT HADAMARD PRODUCTS
}

\author{
By KazUya TOHGE
}

\section{§ 0. Introduction.}

In [1] A. Hinkkanen proposed the following problem:

2.65. Since the knowledge of the zeros of an entire function $f$ leaves an unknown factor, $e^{h}$ say, in the Hadamard product for $f$, one can ask if $f$ is determined by the zeros of $f$ and its first few derivatives. Does there exist an integer $k \geqq 2$ such that, if $f$ and $g$ are entire and $f^{(n)} / g^{(n)}$ is entire and nonvanishing for $0 \leqq n \leqq k$, then $f / g$ is constant unless

$$
f(z)=e^{a z+b}, g(z)=e^{c z+d} \quad \text { or } \quad f(z)=A\left(e^{a z}-b\right), g(z)=B\left(e^{-a z}-b^{-1}\right) \text { ? }
$$

The proposer has shown (unpublished) that $k=2$ will do in certain cases; for example, when $f$ and $g$ have finite order. The example

$$
f(z)=\left(e^{2 z}-1\right) \exp \left(-i e^{z}\right), \quad g(z)=\left(1-e^{-2 z}\right) \exp \left(i e^{-z}\right)
$$

shows that one sometimes needs $k=3$.

One can ask a similar question for meromorphic functions, with the additional possibility that

$$
f(z)=A\left(e^{h(z)}-1\right)^{-1}, \quad g(z)=B\left(1-e^{-h(z)}\right)^{-1}
$$

for any non-constant entire function $h$.

On this problem G. G. Gundersen [3] gave some information about the connection between the unknown factor and the zeros. In this paper we shall investigate this problem when $k=2$ under the restriction on the order $\rho$ of $h$, that is, $\rho<2$. Then we can see that the answer to this problem is yes when $k=4$, and if $k=3$, there is only one possibility in addition.

We assume familiarity with Nevanlinna's theory and with its standard notations (see, [4]).

\section{$\S 1$. Results.}

THEOREM. Suppose that two meromorphic functions $f$ and $g$ of hyper-order less than 2 satisfy the condition that $f^{(n)} / g^{(n)}$ is entire and non-vanishing for $0 \leqq$

Received January 23, 1989; revised July 20, 1989 
$n \leqq 2$. Then $\mathrm{f} / \mathrm{g}$ reduces to a constant unless

$$
\begin{gathered}
f(z)=e^{a z+b}, \quad g(z)=e^{c z+d} ; \\
f(z)=A\left(e^{a z}-c\right), \quad g(z)=B\left(e^{-a z}-c^{-1}\right) ; \\
f(z)=A\left(e^{h(z)}-1\right)^{-1}, \quad g(z)=B\left(1-e^{-h(z)}\right)^{-1}
\end{gathered}
$$

for any nonconstant entire function $h$ of order $<2$;

$$
\begin{gathered}
f(z)=A \exp \left\{c\left(e^{a z+b}-a z\right)\right\}, \quad g(z)=B \exp \left\{c\left(e^{-(a z+b)}+a z\right)\right\} ; \\
f(z)=A\left(e^{a z+b}-1\right)^{-2} \exp \left(2 a z-e^{-(a z+b)}\right), \\
g(z)=B\left(e^{a z+b}-1\right)^{-2} \exp \left(-2 e^{a z+b}\right) ; \\
f(z)=A \exp \left\{\int_{0}^{z} \frac{a c\left(e^{a \zeta+b}-1\right) d \zeta}{(1-c)(a \zeta+b+2 k \pi i)}\right\} \\
g(z)=B \exp \left\{\int_{0}^{z} \frac{a\left(1-e^{-(a \zeta+b)}\right) d \zeta}{(1-c)(a \zeta+b+2 k \pi i)}\right\}
\end{gathered}
$$

with an integer $k$ and $c \neq 1$;

$$
\begin{aligned}
& f(z)=A(a z+b+2 k \pi i) \exp \left\{\int_{0}^{z} \frac{a\left(e^{a \zeta+b}-1\right) d \zeta}{(1-c)(a \zeta+b+2 k \pi i)}\right\}, \\
& g(z)=B(a z+b+2 k \pi i) \exp \left\{\int_{0}^{z} \frac{a c\left(1-e^{-(a \zeta+b)}\right) d \zeta}{(1-c)(a \zeta+b+2 k \pi i)}\right\}
\end{aligned}
$$

with an integer $k$ and $c \neq 1$;

$$
\begin{aligned}
& f(z)=A H(z) \exp \left[-\int_{0}^{z} H(\zeta)^{-1}\left\{H^{\prime}(\zeta)+c e^{-m(a \zeta+b)}\left(\frac{e^{a \zeta+b}-1}{L(\zeta)}\right)^{m}\right\} d \zeta\right], \\
& g(z)=B H(z) \exp \left[-\int_{0}^{z} H(\zeta)^{-1}\left\{H^{\prime}(\zeta)+\left(\frac{e^{a \zeta+b}-1}{L(\zeta)}\right)^{m}\right\} d \zeta\right]
\end{aligned}
$$

with an integer $m(\geqq 2)$ and $c \neq 1$, provided that $H$ and $L$ are entire functions of order at most 1 , vanishing only at zeros of $e^{m(a z+b)}-c$ and of $e^{a z+b}-1$, respectively, and satisfy

$$
H^{\prime}(z) L(z)+m H(z) L^{\prime}(z)=\left(\frac{e^{a z+b}-1}{L(z)}\right)^{m-1} e^{-(m-1)(a z+b)}\left(e^{(m-1)(a z+b)}-c\right) ;
$$

or possibly,

$$
f(z)=H(z) L(z)^{k} \exp (N(z)), \quad g(z)=H(z) L(z)^{k} \exp (M(z)),
$$

where entire functions $H$ and $L$ are given by

$$
(H(z) L(z))^{\prime}\left(e^{c z+d}-1\right)-a e^{c z+d} H(z) L(z)=e^{a z+b} e^{c z+d}-1 .
$$


Further $H$ vanishes only at one-points of $e^{a z+b}$ excepting those of $e^{c z+d}$, and $L$ vanishes at all the one-points common to $e^{a z+b}$ and $e^{c z+d}$. Also $M$ and $N$ are entire functions satisfying

$$
M^{\prime}=\frac{1-H^{\prime} L-k H L^{\prime}}{H L} \text { and } \quad N^{\prime}=\frac{e^{a z+b}-H^{\prime} L-k H L^{\prime}}{H L} .
$$

Here $H L$ is not zero-free, and if $L$ has never a zero we may chose it the constant 1. While otherwise, $k$ is an integer different from $0, \pm 1,-2$ and -3 , and then $-a / c$ is a rational number equal to $(k-1) /(k+1)$ but a positive integer.

In any case, $A, B, a, b, c$ and $d$ are constants with $A \cdot B \cdot a \cdot c \neq 0$.

COROLlARY. In the above theorem, if we suppose additionally that $f^{\prime \prime \prime} / g^{\prime \prime \prime}$ is also entire and non-vanishing, then the possible pairs are those of (1), (2), (3) as in the theorem and

$$
f(z)=A \exp \left(e^{a z+b}\right), \quad g(z)=B \exp \left(e^{-(a z+b)}\right) .
$$

The pair (10) is obtained from the possibility (8) by setting $L(z)=e^{a z+b}-1$, $c=-1$ and $m=2$. Also the example given by Hinkkanen is contained in (8) with $a=1, e^{b}=i, c=-1, m=2$ and $L(z) \equiv 1$, thus

$$
H(z)=i\left(e^{2 z}-1\right) e^{-z} .
$$

The author, however, do not know whether there indeed exist entire functions $H$ and $L$ as in (9).

In this theorem it is sufficient for our proof that $f / g=e^{\alpha}$ say, has hyperorder less than 2, that is, the order of $\alpha$ is less than 2 . On the restriction we can say slightly more that Theorem still holds when it is of order 2 and minimal type. Then the function $h$ in (3) should be so. Without this restriction our method, Lemma 1, does not work in the case where

$$
\limsup _{\substack{r \rightarrow \infty \\(r \notin E)}} \frac{m\left(r, e^{h}\right)+m\left(r, e^{k}\right)}{m\left(r, \alpha^{\prime}\right)}<\infty
$$

for $e^{h}=\frac{f^{\prime}}{f} / \frac{g^{\prime}}{g} \not \equiv 1$ and $e^{k}=\frac{f^{\prime \prime}}{f^{\prime}} / \frac{g^{\prime \prime}}{g^{\prime}} \not \equiv 1$. In this section $E$ denotes a set of finite linear measure, which is not always the same. Then we can see

$$
\underset{\substack{r \rightarrow \infty \\(r \notin E)}}{\limsup } \frac{\bar{N}(r, g)+\bar{N}(r, 1 / g)+\bar{N}\left(r, 1 / g^{\prime}\right)+N_{0}\left(r, 1 / g^{\prime}\right)+N_{0}\left(r, 1 / g^{\prime \prime}\right)}{m\left(r, \alpha^{\prime}\right)}<\infty .
$$

Here in $N_{0}\left(r, 1 / \psi^{\prime}\right)$ only zeros of $\psi^{\prime}(z)$ not corresponding to the multiple zeros of $\psi(z)$ are to be considered. The following is such a pair satisfying the assumptions of our theorem apart from the restriction on the order of $\alpha$,

$$
f(z)=\exp \left\{c \int^{z}\left(e^{G(\zeta)}-1\right) d \zeta\right\} \text { and } g(z)=\exp \left\{c \int^{z}\left(1-e^{-G(\zeta)}\right) d \zeta\right\}
$$


where $G$ is any entire function. In fact, then $e^{h(z)}=e^{k(z)}=e^{G(z)}$ and

$$
\alpha^{\prime}(z)=c e^{-G(z)}\left(e^{G(z)}-1\right)^{2},
$$

therefore $m\left(r, \alpha^{\prime}\right) \geqq(2+o(1)) m\left(r, e^{G}\right)(r \notin E)$, unless $G$ is a constant.

\section{$\S 2$. Preliminaries.}

To obtain our results we shall make proper use of two tools according to the order of the function $\alpha^{\prime}=f^{\prime} / f-g^{\prime} / g$. One is the so-called Borel's unicity theorem, or impossibility of Borel's identity, which is expressed as in Lemmas 1 and 2. The other is an elementary fact in the theory of Ordinary differential equations: for functions $f_{1}$ and $f_{2}$ holomorphic in a simply connected domain $D$, the solution of Riccati's equation

is given by

$$
w^{\prime}(z)=f_{1}(z) w(z)+f_{2}(z)[w(z)]^{2}
$$

$$
\begin{aligned}
w(z) & =w\left(z ; z_{0}, 1 / c_{0}\right) \\
& =\left[c_{0} \exp \left\{-\int_{z_{0}}^{z} f_{1}(\zeta) d \zeta\right\}-\int_{z_{0}}^{z} f_{2}(\zeta) \exp \left\{-\int_{\zeta}^{z} f_{1}(\xi) d \xi\right\} d \zeta\right]^{-1},
\end{aligned}
$$

where $z_{0} \in D$ and $c_{0}$ is a nonzero constant (see, for instance [5]).

LEMmA 1 (Hiromi and Ozawa [6]). Let $a_{0}(z), a_{1}(z), \cdots, a_{n}(z)$ be meromorphic functions and let $g_{1}(z), \cdots, g_{n}(z)$ be entire functions. Further suppose that

$$
T\left(r, a_{j}\right)=o\left\{\sum_{\nu=1}^{n} m\left(r, e^{g_{\nu}}\right)\right\}, \quad j=0,1, \cdots, n
$$

holds outside a set of finite linear measure. If an identity

$$
\sum_{\nu=1}^{n} a_{\nu}(z) e^{g_{\nu}(z)}=a_{0}(z)
$$

holds, then we have an identity

$$
\sum_{\nu=1}^{n} c_{\nu} a_{\nu}(z) e^{g_{\nu}(z)}=0
$$

where the constants $c_{\nu}, \nu=1, \cdots, n$, are not all zero.

LEMMA 2 (Gross [2; p. 108, Lemma 5.1]). Let $a_{i}(z)$ be entire of finite order $\leqq \rho$. Let $g_{i}(z)$ be entire, and let $g_{i}(z)-g_{j}(z)(i \neq j)$ be a transcendental function or polynomial of degree greater than $\rho$. Then

$$
\sum_{i=1}^{n} a_{i}(z) e^{g_{i}(z)}=a_{o}(z)
$$

holds only when 


$$
a_{o}(z)=a_{1}(z)=\cdots=a_{n}(z) \equiv 0 .
$$

Also we need the following unicity lemma:

LEMMA 3 (Osgood and Yang [7]). Let $P(z), Q(z)$ be two nonconstant polynomials of the same degree. If

$$
f(z)=\frac{e^{P(z)}-1}{e^{Q(z)}-1}
$$

is entire then

$$
P(z)=m(Q(z))+2 n \pi i,
$$

where $m, n$ are integers.

\section{$\S 3$. Proof of Theorem and Corollary.}

By the assumption let us set

$$
\begin{aligned}
& f(z)=g(z) e^{\alpha(z)} \\
& f^{\prime}(z)=g^{\prime}(z) e^{\beta(z)}, \quad \text { and } \\
& f^{\prime \prime}(z)=g^{\prime \prime}(z) e^{\gamma(z)},
\end{aligned}
$$

where $\alpha, \beta$ and $\gamma$ are entire functions of order less than 2 .

There is nothing to prove if $\alpha$ is a constant. Also if $\beta$ is a constant, then $f(z)=C g(z)+D$ with constants $C=e^{\beta}$ and $D$. Suppose that $D \neq 0$. Then this together with (3.0.1) gives

$$
g(z)=\frac{D}{e^{\alpha(z)}-C} \quad \text { and } \quad f(z)=\frac{D}{1-C e^{-\alpha(z)}},
$$

where $\alpha$ is a nonconstant entire function. This is found in (3) of our theorem.

Now we suppose that neither $\alpha^{\prime}$ nor $\beta^{\prime}$ vanishes identically. Then differentiation gives

$$
\frac{g^{\prime}(z)}{g(z)}=\frac{\alpha^{\prime}(z)}{e^{\beta(z)-\alpha(z)}-1}
$$

and

$$
\frac{g^{\prime \prime}(z)}{g^{\prime}(z)}=\frac{\beta^{\prime}(z)}{e^{\gamma(z)-\beta(z)}-1}
$$

from (3.0.1), (3.0.2) and (3.0.3). Further differentiation and substitution of (3.0.4) and (3.0.5) give the initial identity

$$
\left(\alpha^{\prime \prime}+\alpha^{\prime 2}\right) e^{-\alpha+2 \beta-\gamma}-\left\{\alpha^{\prime \prime}-\alpha^{\prime}\left(\beta^{\prime}-\alpha^{\prime}\right)\right\} e^{-\alpha+\beta}-\left\{\alpha^{\prime \prime}+\alpha^{\prime}\left(\beta^{\prime}-\alpha^{\prime}\right)\right\} e^{\beta-\gamma}=\alpha^{\prime 2}-\alpha^{\prime \prime} .
$$

Now we shall show that functions $\beta-\alpha$ and $\beta-\gamma$ must be linear. 


\section{§ 3.1. Linearity of $\beta-\alpha$ and $\beta-\gamma$.}

Case 1: $\beta-\alpha$ is not linear.

Let $\beta-\alpha$ be denoted by $h$. Then (3.0.6) becomes

$$
\left(\alpha^{\prime \prime}+\alpha^{\prime 2}\right) e^{2 h+\alpha-\gamma}-\left(\alpha^{\prime \prime}-h^{\prime} \alpha^{\prime}\right) e^{h}-\left(\alpha^{\prime \prime}+h^{\prime} \alpha^{\prime}\right) e^{h+\alpha-\gamma}=\alpha^{\prime 2}-\alpha^{\prime \prime} .
$$

We note that neither $\alpha^{\prime \prime}+\alpha^{2}$ nor $\alpha^{\prime \prime}-\alpha^{\prime 2}$ vanishes identically for our function $\alpha$ is nonconstant entire. Also we have two estimates

$$
m\left(r, \alpha^{\prime}\right)=S\left(r, e^{h}\right) \text { and } m\left(r, h^{\prime}\right)=S\left(r, e^{h}\right)
$$

by our assumption on the order of $\alpha$ and the theorem on logarithmic derivatives (see, [4]).

If $\alpha^{\prime \prime}-h^{\prime} \alpha^{\prime} \equiv 0$, then (3.1.1) becomes

$$
\left(\alpha^{\prime \prime}+\alpha^{\prime 2}\right) e^{2 h+\alpha-\gamma}-2 \alpha^{\prime \prime} e^{h+\alpha-\gamma}=\alpha^{\prime 2}-\alpha^{\prime \prime}
$$

and while $\alpha^{\prime \prime}+h^{\prime} \alpha^{\prime} \equiv 0$,

$$
\left(\alpha^{\prime \prime}+\alpha^{2}\right) e^{2 h+\alpha-\gamma}-2 \alpha^{\prime \prime} e^{h}=\alpha^{2}-\alpha^{\prime \prime} .
$$

Due to (3.1.2) Lemma 1 leads then each identity to

$$
C\left(\alpha^{\prime \prime}+\alpha^{2}\right) e^{h}=2 \alpha^{\prime \prime} \quad \text { and } \quad\left(\alpha^{\prime \prime}+\alpha^{\prime 2}\right) e^{2 h+\alpha-\gamma}=2 C \alpha^{\prime \prime} e^{h}
$$

for a nonzero constant $C$, respectively. The former gives a contradiction by (3.1.2). For the latter equation, by substituting it into (3.1.3) we have

$$
2(C-1) \alpha^{\prime \prime} e^{h}=\alpha^{2}-\alpha^{\prime \prime},
$$

which is also a contradiction.

An application of the lemma to (3.1.1) gives an identity

$$
C_{1}\left(\alpha^{\prime \prime}+\alpha^{\prime 2}\right) e^{h+\alpha-\gamma}+C_{3}\left(\alpha^{\prime \prime}+h^{\prime} \alpha^{\prime}\right) e^{\alpha-\gamma}=-C_{2}\left(\alpha^{\prime \prime}-h^{\prime} \alpha^{\prime}\right),
$$

where the constants $C_{\nu}$ are not all zero. Then $C_{1}=0$ implies $C_{2} \cdot C_{3} \neq 0$ and

$$
\alpha^{\prime \prime}-h^{\prime} \alpha^{\prime}=C\left(\alpha^{\prime \prime}+h^{\prime} \alpha^{\prime}\right) e^{\alpha-\gamma}, \quad C=-C_{3} / C_{2} .
$$

On substituting this into (3.1.1) we obtain

$$
\left(\alpha^{\prime \prime}+\alpha^{2}\right) e^{2 h+\alpha-\gamma}-(C+1)\left(\alpha^{\prime \prime}+h^{\prime} \alpha^{\prime}\right) e^{h+\alpha-\gamma}=\alpha^{\prime 2}-\alpha^{\prime \prime} .
$$

Here a simple analysis shows $C+1 \neq 0$. Thus a further application of the lemma leads this to the result

$$
\left(\alpha^{\prime \prime}+\alpha^{2}\right) e^{h}=D\left(\alpha^{\prime \prime}+h^{\prime} \alpha^{\prime}\right)
$$

with a nonzero constant $D$. The estimation 


$$
m\left(r, e^{h}\right) \leqq m\left(r, e^{2 h+\alpha-r}\right)+m\left(r, e^{h+\alpha-r}\right)+O(1)
$$

makes this possible. The result is however impossible due to (3.1.2). Also $C_{3}=0$ gives $C_{1} \cdot C_{2} \neq 0$ and

$$
\left(\alpha^{\prime \prime}-h^{\prime} \alpha^{\prime}\right) e^{h}=C\left(\alpha^{\prime \prime}+\alpha^{\prime 2}\right) e^{2 h+\alpha-\gamma}, \quad C=-C_{1} / C_{2} .
$$

Again by substituting this into (3.1.1) we have

$$
(1-C)\left(\alpha^{\prime \prime}+\alpha^{\prime 2}\right) e^{2 h+\alpha-\gamma}-\left(\alpha^{\prime \prime}+h^{\prime} \alpha^{\prime}\right) e^{h+\alpha-\gamma}=\alpha^{2}-\alpha^{\prime \prime} .
$$

Then we must take $C=1$ and thus

$$
\left(\alpha^{\prime \prime}+h^{\prime} \alpha^{\prime}\right) e^{h+\alpha-\gamma}=\alpha^{\prime \prime}-\alpha^{\prime 2} \text { and }\left(\alpha^{\prime \prime}+\alpha^{\prime 2}\right) e^{h+\alpha-\gamma}=\alpha^{\prime \prime}-h^{\prime} \alpha^{\prime} .
$$

Hence we arrive at the results $\alpha^{\prime}=h^{\prime}$ and $\left(e^{h+\alpha-\gamma}-1\right) \alpha^{\prime \prime}=-\left(e^{h+\alpha-\gamma}+1\right) \alpha^{\prime 2}$, since $h^{\prime}+\alpha^{\prime}=\beta^{\prime} \neq 0$. Then the property of exponential functions and entireness of $\alpha^{\prime}(\not \equiv 0)$ permit only to get $e^{h+\alpha-\gamma} \equiv-1$ and $\alpha^{\prime \prime} \equiv 0$, which is however impossible now. Thus we may apply Lemma 1 to (3.1.4) and get a contradiction again.

Case 2: $-\alpha+\beta$ is of the form $A z+B, A$ and $B \in C$, with $e^{A z+B} \not \neq 1$.

Then our initial identity (3.0.6) becomes

$$
\left(\alpha^{\prime \prime}+\alpha^{\prime 2}\right) e^{A z+B} e^{\beta-\gamma}-\left(\alpha^{\prime \prime}-A \alpha^{\prime}\right) e^{A z+B}-\left(\alpha^{\prime \prime}+A \alpha^{\prime}\right) e^{\beta-\gamma}=\alpha^{2}-\alpha^{\prime \prime} .
$$

Now we suppose that $\beta-\gamma$ is a non-linear entire function. Then the estimates

$$
m\left(r, \alpha^{\prime}\right)=S\left(r, e^{\beta-r}\right) \text { and } m\left(r, e^{A z+B}\right)=S\left(r, e^{\beta-r}\right)
$$

reduce $(3.1 .5)$ to the result

$$
\left(\alpha^{\prime \prime}+\alpha^{2}\right) e^{A z+B}-\left(\alpha^{\prime \prime}+A \alpha^{\prime}\right)=\left(\alpha^{\prime \prime}-A \alpha^{\prime}\right) e^{A z+B}-\left(\alpha^{\prime \prime}-\alpha^{\prime 2}\right) \equiv 0 .
$$

Then we have $\alpha^{\prime} \equiv \pm A$ and $A^{2} e^{A z+B} \equiv \pm A^{2}$. This is impossible since $\alpha^{\prime} \not \equiv$.

Hence we need only to discuss Identity (3.1.5) when $\beta-\gamma$ is also of the form $C z+D, C, D \in C$, with $e^{C z+D} \not \equiv 1$. Then our identity is

$$
\left(\alpha^{\prime \prime}+\alpha^{\prime 2}\right) e^{(A+C) z+(B+D)}-\left(\alpha^{\prime \prime}-A \alpha^{\prime}\right) e^{A z+B}-\left(\alpha^{\prime \prime}+A \alpha^{\prime}\right) e^{C z+D}=\alpha^{2}-\alpha^{\prime \prime} .
$$

There is a difference in methods according to the growth of $\alpha^{\prime}$. Therefore we distinguish the cases whether $\alpha^{\prime}$ is of order less than one, or not.

\section{§3.2. The case where the order of $\alpha^{\prime}$ is less than one.}

Then the method that we make use of is Borel's unicity theorem represented as Lemma 2. To do this we have four possibilities to be considered in (3.1.6). The first one is that $A=0$. Then (3.1.6) becomes

$$
\left\{\left(e^{B}-1\right) \alpha^{\prime \prime}+e^{B} \alpha^{\prime 2}\right\} e^{C z+D}=\left(e^{B}-1\right) \alpha^{\prime \prime}+\alpha^{\prime 2} .
$$


It now follows either $C=0$ or

$$
\left(e^{B}-1\right) \alpha^{\prime \prime}+e^{B} \alpha^{\prime}=\left(e^{B}-1\right) \alpha^{\prime \prime}+\alpha^{\prime 2} \equiv 0
$$

from the present assumption. The latter result gives $e^{B}=1$. This is however outside our observation. While $C=0$ gives the identity

$$
\left(e^{B}-1\right)\left(e^{D}-1\right) \alpha^{\prime \prime}+\left(e^{B+D}-1\right) \alpha^{2} \equiv 0 .
$$

The entireness of $\alpha^{\prime}$ shows that $e^{B+D}=1$ and $\alpha^{\prime}$ is a constant. Then the equations (3.0.4) and (3.0.1) imply the result (1).

Next we suppose that $A \neq 0$ and $C=0$. Then

$$
\left\{\left(e^{D}-1\right) \alpha^{\prime \prime}+e^{D} \alpha^{2}+A \alpha^{\prime}\right\} e^{A z+B}=\left(e^{D}-1\right) \alpha^{\prime \prime}+\alpha^{\prime 2}+A e^{D} \alpha^{\prime},
$$

and the assumption leads this to the result

$$
\left(e^{D}-1\right) \alpha^{\prime \prime}+e^{D} \alpha^{2}+A \alpha^{\prime}=\left(e^{D}-1\right) \alpha^{\prime \prime}+\alpha^{\prime 2}+A e^{D} \alpha^{\prime} \equiv 0 .
$$

Hence we have $\alpha^{\prime} \equiv A$ and $e^{D}=-1$. Then (3.0.4) and (3.0.1) give the pair $(2)$.

In the third possibility, $A+C=0$ and $A \neq 0$, the identity (3.1.6) becomes

$$
\left(\alpha^{\prime \prime}-A \alpha^{\prime}\right) e^{A z+B}+\left(\alpha^{\prime \prime}+A \alpha^{\prime}\right) e^{B+D} e^{-(A z+B)}=\left(e^{B+D}+1\right) \alpha^{\prime \prime}+\left(e^{B+D}-1\right) \alpha^{2} .
$$

An application of Lemma 2 leads us to the conclusion

$$
\alpha^{\prime \prime}-A \alpha^{\prime}=\alpha^{\prime \prime}+A \alpha^{\prime}=\left(e^{B+D}+1\right) \alpha^{\prime \prime}+\left(e^{B+D}-1\right) \alpha^{2} \equiv 0,
$$

which is evidently impossible.

Finally if $A=C \neq 0$, then

$$
\left(\alpha^{\prime \prime}+\alpha^{\prime 2}\right) e^{B+D} e^{2 A z}-\left\{\left(e^{B}+e^{D}\right) \alpha^{\prime \prime}-\left(e^{B}-e^{D}\right) A \alpha^{\prime}\right\} e^{A z}=\alpha^{2}-\alpha^{\prime \prime},
$$

which implies by the lemma

$$
\alpha^{\prime \prime}+\alpha^{\prime 2}=\left(e^{B}+e^{D}\right) \alpha^{\prime \prime}-\left(e^{B}-e^{D}\right) A \alpha^{\prime}=\alpha^{2}-\alpha^{\prime \prime} \equiv 0 .
$$

This is again impossible. Therefore the application of Lemma 2 to (3.1.6) shows that the identity is impossible due to the similar reason. The observation is now completed on the present assumption.

\section{$\S 3.3$. The case where $\alpha^{\prime}$ is of order $\rho$ for $1 \leqq \rho<2$.}

Here we regard Equation (3.1.6) as

$$
\left(e^{A z+B}-1\right)\left(e^{C z+D}-1\right) \alpha^{\prime \prime}+\left(e^{A z+B} e^{C z+D}-1\right) \alpha^{2}+A\left(e^{A z+B}-e^{C z+D}\right) \alpha^{\prime}=0,
$$

that is, we consider that $\alpha^{\prime}$ is an entire solution of Riccati's equation

$$
w^{\prime}+\frac{\left(e^{A z+B} e^{C z+D}-1\right)}{\left(e^{A z+B}-1\right)\left(e^{C z+D}-1\right)} w^{2}+A \frac{\left(e^{A z+B}-e^{C z+D}\right)}{\left(e^{A z+B}-1\right)\left(e^{C z+D}-1\right)} w=0 .
$$


Hence by the fact mentioned in $\S 2$, a solution of (3.3.2) is given by

$$
\begin{aligned}
w(z)= & w\left(z ; z_{0}, 1 / C_{0}\right) \\
= & {\left[C_{0} \exp \left\{\int_{z_{0}}^{z} A \frac{e^{A \zeta+B}-e^{C \zeta+D}}{\left(e^{A \zeta+B}-1\right)\left(e^{C \zeta+D}-1\right)} d \zeta\right\}\right.} \\
& \left.+\int_{z_{0}}^{z} \frac{e^{A \zeta+B} e^{C \zeta+D}-1}{\left(e^{A \zeta+B}-1\right)\left(e^{C \zeta+D}-1\right)} \exp \left\{\int_{\zeta}^{z} A \frac{e^{A \xi+B}-e^{C \xi+D}}{\left(e^{A \xi+B}-1\right)\left(e^{C \xi+D}-1\right)} d \xi\right\} d \zeta\right]^{-1}
\end{aligned}
$$

possibly apart from zeros of $e^{A z+B}-1$ and of $e^{C z+D}-1$. In the strict sense, the zeros of $e^{A z+B}-1$ and of $e^{C z+D}-1$ are all joined to the point at infinity by cuts which have no finite points in common, and as our domain $D$ we take the complex plane less the cuts. And let $z_{0} \in D$ and also choose $C_{0}(\neq 0)$ arbitrarily. We note that we shall take suitable branches as the occasion demands.

There are three cases to be separated in our observation, that is, (i) $A=0$, (ii) $C=0$ and $A \neq 0$, and (iii) otherwise. In the case (i), (3.3.3) becomes

$$
w(z)=\left\{C_{0}+\int_{z_{0}}^{z} \frac{\left(e^{B} e^{C \zeta+D}-1\right) d \zeta}{\left(e^{B}-1\right)\left(e^{C \zeta+D}-1\right)}\right\}^{-1}
$$

with $e^{B} \neq 1$. A simple analysis shows that this does not represent any nonconstant entire function. While the assumption (ii) leads to

$$
w(z)=A d(d-1)\left(e^{A z+B}-1\right)\left\{C_{1} e^{d(A z+B)}-d^{2} e^{A z+B}+(d-1)^{2}\right\}^{-1}
$$

for $d=e^{D} /\left(e^{D}-1\right)$ and a constant $C_{1}$ depending on $z_{0}, C_{0}$ and $d$. We require that every zero of the denominator should be cancelled by that of the numerator, since $w(z)$ must be entire. Easily we see that $C_{1} \neq 0$. Hence we obtain $C_{1}=$ $(2 d-1) e^{2 m d \pi \imath}$ for some integer $m$. Then we can show by Lemma 3 that $d$ must be a rational number, even so $w(z)$ in (3.3.4) represents no entire function.

In the general case, a calculation gives

$$
w(z)=e^{2 l \pi \imath} \frac{e^{A z+B}-1}{\left(e^{C z+D}-1\right)^{A / C}}\left\{C_{1}+\int_{z_{0}}^{z} \frac{e^{A \zeta+B} e^{C \zeta+D}-1}{\left(e^{C \zeta+D}-1\right)^{A / C+1}} d \zeta\right\}^{-1} \quad(z \in D)
$$

with an integer $l$ and the constant

$$
C_{1}=C_{0} e^{2 n \pi(A / C) \imath}\left(e^{A z_{0}+B}-1\right)\left(e^{C z_{0}+D}-1\right)^{-A / C}, \quad n \text { an integer. }
$$

Suppose that $A+C=0$, then

$$
w(z)=-e^{-(A z+B)}\left(e^{A z+B}-1\right)\left(e^{A z+B}-e^{B+D}\right)\left\{C_{1}+\left(e^{B+D}-1\right)\left(z-z_{0}\right)\right\}^{-1} .
$$

If $e^{B+D}=1, C_{1} \neq 0$ and we have the entire function

$$
\alpha^{\prime}(z)=K e^{-(A z+B)}\left(e^{A z+B}-1\right)^{2}, \quad K \in C \backslash\{0\},
$$

thus by (3.0.4) 


$$
\frac{g^{\prime}(z)}{g(z)}=K\left(1-e^{-(A z+B)}\right) \text {. }
$$

Hence we can get the pair (4). Calculation shows that this pair does not satisfy the further condition required in Corollary.

While if $e^{B+D}$ is different from one, the constant $C_{1}-\left(e^{B+D}-1\right) z_{0}$ must be equal to either of

$$
(2 k \pi i+B)\left(e^{B+D}-1\right) / A \text { and }(2 k \pi i-D)\left(e^{B+D}-1\right) / A
$$

for an integer $k$. Then the function $w^{\prime}(z)$ represents our function $\alpha^{\prime}(z)$,

$$
\frac{A e^{-(A z+B)}\left(e^{A z+B}-1\right)\left(e^{A z+B}-e^{B+D}\right)}{\left(1-e^{B+D}\right)(A z+B+2 k \pi i)} \text { and } \frac{A e^{-(A z+B)}\left(e^{A z+B}-1\right)\left(e^{A z+B}-e^{B+D}\right)}{\left(1-e^{B+D}\right)\{A z+B-(B+D)+2 k \pi i\}},
$$

respectively. By (3.0.4) and (3.0.1) the former case implies the result (7) with the help of (3.0.5). And for the latter we have the result (6) similarly by (3.0.4) and (3.0.1). In each case their third order derivatives do not have the property required in Corollary.

Next we put $A=C$. Then we can see that $e^{B}+e^{D}=0$ and

$$
w(z)=-A\left(e^{A z+B}-1\right)\left\{A\left(C_{2}-z\right)\left(e^{A z+B}-1\right)-2\right\}^{-1}
$$

for some constant $C_{2}$. Evidently this can not be any entire function. Hence we shall proceed to the general case that is the main part of this proof.

\section{§ 3.4. Discussion about the monodromy of the function $w(z)$.}

Recall that we consider an entire function $\alpha$ satisfying the equation (3.3.1) and note that for a zero of $e^{A z+B}-1, z_{1}$ say,

$$
\left(e^{C z_{1}+D}-1\right) \alpha^{\prime}\left(z_{1}\right)\left\{\alpha^{\prime}\left(z_{1}\right)-A\right\}=0,
$$

and for a zero $z_{1}$ of $e^{C z+D}-1$,

$$
\left(e^{A z_{1}+B}-1\right) \alpha^{\prime}\left(z_{1}\right)\left\{\alpha^{\prime}\left(z_{1}\right)+A\right\}=0 .
$$

Now we require that one of the solutions given by (3.3.5) in $D$ should be the $\alpha^{\prime}$, a single-valued and regular function in the whole plane. In fact the one-points of $e^{A z+B}$ are regular points of them. For our purpose therefore it is sufficient to impose the following monodromy condition on the $w(z)$ : Let $z^{\prime}$ be any point in a neighbourhood of a one-point $z_{1}$ of $e^{C z+D}$ and $\Gamma=\Gamma\left(z^{\prime} ; z_{1}\right)$ any sufficiently small circular loop at $z^{\prime}$ with center $z_{1}$. Then the value $w\left(z^{\prime}\right)$ at the initial point of $\Gamma$ should coincide with the value $w^{*}\left(z^{\prime}\right)$ at the terminal point.

Evidently as $z$ describes $\Gamma, e^{C z+D}-1$ winds the origin once with the same (positive) direction. Hence we have 


$$
\begin{aligned}
& w^{*}\left(z^{\prime}\right)= \\
& \frac{e^{2 \pi l(A / C) \imath} e^{-2 \pi(A / C) 2}\left(e^{A z^{\prime}+B}-1\right)\left(e^{C z^{\prime}+D}-1\right)^{-A / C}}{C_{1}+\int_{z_{0}}^{z^{\prime}}\left(e^{A \zeta+B} e^{C \zeta+D}-1\right)\left(e^{C \zeta+D}-1\right)^{-A / C-1} d \zeta+\int_{\Gamma}\left(e^{A \zeta+B} e^{C \zeta+D}-1\right)\left(e^{C \zeta+D}-1\right)^{-A / C-1} d \zeta} .
\end{aligned}
$$

The condition $w\left(z^{\prime}\right)=w^{*}\left(z^{\prime}\right)$ is thus equivalent to the representation

$$
\begin{aligned}
& \int_{\Gamma}\left(e^{A \zeta+B} e^{C \zeta+D}-1\right)\left(e^{C \zeta+D}-1\right)^{-A / C-1} d \zeta \\
& \quad=\left(e^{-2 \pi(A / C) \imath}-1\right)\left\{C_{1}+\int_{z_{0}}^{z^{\prime}}\left(e^{A \zeta+B} e^{C \zeta+D}-1\right)\left(e^{C \zeta+D}-1\right)^{-A / C-1} d \zeta\right\} .
\end{aligned}
$$

Suppose $e^{-2 \pi(A / C) \imath} \neq 1$. Then

$$
w\left(z^{\prime}\right)=\frac{e^{2 l \pi(A / C) \imath}\left(e^{-2 \pi(A / C) \imath}-1\right)\left(e^{A z^{\prime}+B}-1\right)\left(e^{C z^{\prime}+D}-1\right)^{-A / C}}{\int_{\Gamma}\left(e^{A \zeta+B} e^{C \zeta+D}-1\right)\left(e^{C \zeta+D}-1\right)^{-A / C-1} d \zeta}
$$

and we shall discuss the "value" $w\left(z_{1}\right)$.

Firstly suppose $e^{A z_{1}+B}=1$ as well as $e^{C z_{1}+D}=1$. Then we can see that for $z^{\prime}$ near $z_{1}$,

$$
\begin{aligned}
& \int_{\Gamma}\left(e^{A \zeta+B} e^{C \zeta+D}-1\right)\left(e^{C \zeta+D}-1\right)^{-A / C-1} d \zeta \\
& \quad=e^{2 n \pi(A / C) \imath} \frac{C+A}{C-A} C^{-A / C} \rho^{-A / C+1} e^{\imath(1-A / C) \theta}\left(e^{-2 \pi(A / C) \imath}-1\right)\{1+O(\rho)\},
\end{aligned}
$$

and

$$
\begin{aligned}
& \left(e^{-2 \pi(A / C) \imath}-1\right)\left(e^{A z^{\prime}+B}-1\right)\left(e^{C z^{\prime}+D}-1\right)^{-A / C} \\
& \quad=e^{2 n \pi(A / C) \imath} A C^{-A / C} \rho^{-A / C+1} e^{\imath(1-A / C) \theta}\left(e^{-2 \pi(A / C) \imath}-1\right)\{1+O(\rho)\}
\end{aligned}
$$

for some integer $n$ with $\theta=\operatorname{Arg}\left(z^{\prime}-z_{1}\right)$ and $\rho=\left|z^{\prime}-z_{1}\right|$. Hence

$$
w\left(z^{\prime}\right)=A e^{2 l \pi(A / C) \imath} \frac{C-A}{C+A}\{1+O(\rho)\},
$$

thus $w\left(z_{1}\right)=A e^{2 l \pi(A / C)}(C-A) /(C+A) \neq 0$. Secondly suppose $e^{A z_{1}+B} \neq 1$. Then an analogous observation shows $w\left(z_{1}\right)=-A e^{2 l \pi \imath A / C} \neq 0$. There indeed exists such a point $z_{1}$, since if the function $\left(e^{A z+B}-1\right) /\left(e^{C z+D}-1\right)$ is entire then $A / C$ must be an integer due to Lemma 3. The first remark of this section shows $e^{2 l \pi \imath A / C}=1$, which indicates our choice of branches $Z^{A / C}$ in (3.3.5). Eventually, when $e^{-2 \pi \imath A / C}$ $\neq 1, w(z)$ is different from zero at any zero of $e^{C z+D}-1$. It is also true for a negative $(\leqq-2)$ integer $-A / C$ notwithstanding $e^{-2 \pi i A / C}=1$ in (3.4.1). Hence $w(z)$ may assume the value zero at a zero $z_{1}$ of $e^{C z+D}-1$ only if $-A / C=m$ is a positive $(\geqq 2)$ integer. Now we put

$$
I\left(z_{1} ; z_{0}\right)=C_{1}+\int_{z_{0}}^{z_{1}}\left(e^{A \zeta+B} e^{C \zeta+D}-1\right)\left(e^{C \zeta+D}-1\right)^{-A / C-1} d \zeta,
$$


which is the finite definite value depending only on points $z_{1}$ and $z_{0}$. Also if $e^{A z_{1}+B}=1$,

$$
w(z)=\frac{A C^{m}\left(z-z_{1}\right)^{m+1}\left\{1+O\left(z-z_{1}\right)\right\}}{I\left(z_{1} ; z_{0}\right)+\frac{A+C}{m+1} \cdot C^{m-1} \cdot\left(z-z_{1}\right)^{m+1}\left\{1+O\left(z-z_{1}\right)\right\}}, \text { near } z=z_{1} .
$$

Thus $I\left(z_{1} ; z_{0}\right)=0$ implies $w\left(z_{1}\right)=(m+1) A C /(A+C) \neq 0$. And if $I\left(z_{1} ; z_{0}\right) \neq 0$,

$$
w(z)=\frac{A C^{m}}{I\left(z_{1} ; z_{0}\right)}\left(z-z_{1}\right)^{m+1}\left\{1+O\left(z-z_{1}\right)\right\} \quad \text { near } z=z_{1},
$$

so that $w(z)$ has the zero of multiplicity $m+1$ at $z_{1}$. On the other hand if $e^{A z_{1}+B} \neq 1$, then we can discuss similarly to see that $w\left(z_{1}\right)=m C=-A \neq 0$ for $I\left(z_{1} ; z_{0}\right)=0$ and that $w(z)$ has the zero of multiplicity $m$ at $z_{1}$ for $I\left(z_{1} ; z_{0}\right) \neq 0$.

There is another possibility of its zeros only at zeros of $e^{A z+B}-1$ excepting those of $e^{C z+D}-1$. In any case, at such a zero, $z_{2}$ say,

and

$$
w(z)=A\left\{1+O\left(z-z_{2}\right)\right\} \quad \text { if } \quad I\left(z_{2} ; z_{0}\right)=0
$$

$$
w(z)=\frac{A\left(e^{C z_{2}+D}-1\right)^{-A / C}}{I\left(z_{2} ; z_{0}\right)}\left(z-z_{2}\right)\left\{1+O\left(z-z_{2}\right)\right\},
$$

that is, $w(z)$ has a simple zero otherwise.

Under the above observations we shall distinguish sections according to the value of $-A / C$ to get the representations for $f$ and $g$. The remaining two sections are devoted to this without further investigation of $w(z)$.

\section{$\S 3.5$. The case where $-A / C$ is an integer at least 2 .}

Put $p=-A / C$ and then

$$
\alpha^{\prime}(z)=\frac{e^{-p(C z+D)}\left(e^{p(C z+D)}-e^{B+p D}\right)\left(e^{C z+D}-1\right)^{p}}{-C_{1}+\int_{z_{0}}^{z} e^{-(p-1)(C \zeta+D)}\left(e^{(p-1)(C \zeta+D)}-e^{B+p D}\right)\left(e^{C \zeta+D}-1\right)^{p-1} d \zeta},
$$

by (3.3.5). By our discussion in the previous section it is known that the denominator, $F(z)$ say, can have only a simple zero and zero of multiplicity $p$ possibly at a zero of $e^{p(C z+D)}-e^{B+p D}$ and of $e^{C z+D}-1$, respectively. Then we put

$$
F(z)=H(z) L(z)^{p},
$$

provided that $H$ and $L$ are entire functions assuming the value zero only at zeros of $e^{p(C z+D)}-e^{B+p D}$ and of $e^{C z+D}-1$ satisfying $I\left(z ; z_{0}\right)=0$ with same multiplicity 1 , respectively. We may say that these two functions are of order at most 1. Differentiating this, we have

$$
H^{\prime}(z) L(z)+p H(z) L^{\prime}(z)=\left(\frac{e^{C z+D}-1}{L(z)}\right)^{p-1}\left(e^{(p-1)(C z+D)}-e^{B+p D}\right) e^{-(p-1)(C z+D)} .
$$


There is a difference in our discussion about the constant $b=e^{B+p D}$.

Subcase ; $b \neq 1$.

In this case $H$ and $L$ have no common zero, and

$$
\alpha^{\prime}(z)=\frac{e^{p(C z+D)}-b}{H(z)}\left(\frac{e^{C z+D}-1}{L(z)}\right)^{p} e^{-p(C z+D)} .
$$

By the equation (3.0.4) we have

$$
\frac{g^{\prime}(z)}{g(z)}=-\frac{1}{H(z)}\left(\frac{e^{C z+D}-1}{L(z)}\right)^{p} .
$$

Now we suppose that $H(z)$ has at least a zero, $a$ say. In a neighborhood of $a$,

and (3.5.1) gives

$$
\frac{g^{\prime}(z)}{g(z)}=\frac{1}{z-a} \cdot \frac{-\left(e^{C a+D}-1\right)^{p}}{H^{\prime}(a) L(a)^{p}}\{1+O(z-a)\}
$$

$$
\frac{-\left(e^{C a+D}-1\right)^{p}}{H^{\prime}(a) L(a)^{p}}=1
$$

Hence the residue of $g^{\prime} / g$ is equal to 1 at any zero of $H$, so that $g(z)$ is given by

$$
H(z) \exp (M(z)) \text {. }
$$

Here $M(z)$ is an entire function satisfying

$$
H^{\prime}(z)+M^{\prime}(z) H(z)+\left(\frac{e^{C z+D}-1}{L(z)}\right)^{p}=0 .
$$

In the concrete, we have

$$
\exp (M(z))=K \exp \left[-\int_{0}^{z} \frac{1}{H(\zeta)}\left\{H^{\prime}(\zeta)+\left(\frac{e^{C \zeta+D}-1}{L(\zeta)}\right)^{p}\right\} d \zeta\right], \quad K(\neq 0) \in \boldsymbol{C} .
$$

The desired representations for $f$ and $g$ are

$$
\begin{aligned}
& f(z)=K^{\prime} H(z) \exp \left[-\int_{0}^{z} \frac{1}{H(\zeta)}\left\{H^{\prime}(\zeta)+b e^{-p(C \zeta+D)}\left(\frac{e^{C \zeta+D}-1}{L(\zeta)}\right)^{p}\right\} d \zeta\right] \\
& g(z)=K H(z) \exp \left[-\int_{0}^{z} \frac{1}{H(\zeta)}\left\{H^{\prime}(\zeta)+\left(\frac{e^{C \zeta+D}-1}{L(\zeta)}\right)^{p}\right\} d \zeta\right], \quad K^{\prime}(\neq 0) \in C .
\end{aligned}
$$

It is easy to show that these are also valid if $H(z)$ is different from zero, by taking $H(z) \equiv 1$, for example.

Then the following calculation implies

$$
\frac{f^{\prime \prime \prime}(z)}{g^{\prime \prime \prime}(z)}=b e^{-(p+1)(C z+D)} e^{\alpha(z)} \frac{K(z) H_{1}(z)+J(z)}{K(z) H_{2}(z)+J(z)},
$$

with 


$$
\begin{aligned}
& K(z)=p C-\left(\frac{e^{C z+D}-1}{L(z)}\right)^{p}\left(\frac{e^{p(C z+D)}-b}{H(z)}\right) e^{-p(C z+D)}, \\
& J(z)=p C\left(\frac{e^{C z+D}-1}{L(z)}\right)^{p}\left(e^{C z+D}-1\right) e^{-p(C z+D)}\left(e^{p(C z+D)}+b\right), \\
& H_{1}(z)=C\left(e^{C z+D}-p\right) H(z)+\left(\frac{e^{C z+D}-1}{L(z)}\right)^{p}\left(2-b e^{-(p-1)(C z+D)}-b e^{-p(C z+D)}\right),
\end{aligned}
$$

and

$$
H_{2}(z)=C\left(1-p e^{C z+D}\right) H(z)+\left(\frac{e^{C z+D}-1}{L(z)}\right)^{p}\left(e^{C z+D}+1-2 b e^{-(p-1)(C z+D)}\right) .
$$

In fact, by (3.0.3) we now have

$$
f^{\prime \prime}(z)=b g^{\prime \prime}(z) e^{-(p+1)(C z+D)} e^{\alpha(z)} .
$$

Differentiating this, we obtain the following equation:

$$
f^{\prime \prime \prime}(z)=b e^{-(p+1)(C z+D)} e^{\alpha(z)}\left[g^{\prime \prime \prime}(z)+\left\{-(p+1) C+\alpha^{\prime}(z)\right\} g^{\prime \prime}(z)\right] .
$$

While (3.5.3) together with $F(z)=H(z) L(z)^{p}$ gives

$$
g^{\prime}(z)=-\frac{\left(e^{C z+D}-1\right)^{p}}{F(z)} g(z) .
$$

By differentiation we have from this equation,

where

$$
g^{\prime \prime}(z)=G(z) g^{\prime}(z),
$$

$$
G(z)=\frac{p C e^{C z+D}}{e^{C z+D}-1}-\frac{F^{\prime}(z)}{F(z)}-\frac{\left(e^{C z+D}-1\right)^{p}}{F(z)}
$$

Further we have

$$
g^{\prime \prime \prime}(z)=\left[\frac{G^{\prime}(z)}{G(z)}+G(z)\right] g^{\prime \prime}(z) .
$$

Therefore (3.5.4) and (3.5.6) imply the equation

$$
f^{\prime \prime \prime}(z)=b g^{\prime \prime \prime}(z) e^{-(p+1)(C z+D)} e^{\alpha(z)}\left[1+\frac{G(z)\left\{-(p+1) C+\alpha^{\prime}(z)\right\}}{G(z)^{2}+G^{\prime}(z)}\right] .
$$

Consequently we can obtain the desired representation from (3.5.7), when we take account of the equation

$$
F^{\prime}(z)=\left(e^{C z+D}-1\right)^{p-1}\left(e^{(p-1)(C z+D)}-b\right) e^{-(p-1)(C z+D)}
$$

and use (3.5.5) and (3.5.2).

Under the assumption of Corollary we may put 


$$
e^{r z+s}=\frac{K(z) H_{1}(z)+J(z)}{K(z) H_{2}(z)+J(z)}
$$

for some constants $r$ and $s$. Note that $e^{r z+s} \equiv 1$ implies that $\alpha^{\prime}$ is a constant by (3.5.2) and it is now excluded. Then

$$
e^{r z+s}-1=\frac{K(z)}{J(z)}\left\{H_{1}(z)-e^{r z+s} H_{2}(z)\right\}
$$

and

$$
H_{1}(z)-e^{r z+s} H_{2}(z)=C H(z) h(z)+\left(\frac{e^{C z+D}-1}{L(z)}\right)^{p} k(z)
$$

with

and

$$
h(z)=\left(e^{C z+D}-p\right)-e^{r z+s}\left(1-p e^{C z+D}\right)
$$

$$
k(z)=\left(2-b e^{-(p-1)(C z+D)}-b e^{-p(C z+D)}\right)-e^{r z+s}\left(e^{C z+D}+1-2 b e^{-(p-1)(C z+D)}\right) .
$$

Suppose that $\left(e^{C z+D}-1\right) / L(z)$ has a zero, $z^{*}$ say. Then $J(z)$ has a zero of order at least $p+1$ there and so does $H_{1}(z)-e^{r z+s} H_{2}(z)$ since $K\left(z^{*}\right) \neq 0$. Then $h\left(z^{*}\right)=(1-p)\left(1-e^{r z^{*}+s}\right)=0$ so that $e^{r z^{*}+s}=1$. This means that $H_{1}(z)-e^{r z+s} H_{2}(z)$ has a zero of multiplicity at least $p+2$ there. Since $k\left(z^{*}\right)=2(1-b)\left(1-e^{r z^{*}+s}\right)=0$ and $H\left(z^{*}\right) \neq 0$,

$$
h\left(z^{*}\right)=h^{\prime}\left(z^{*}\right)=\cdots=h^{(p)}\left(z^{*}\right)=0 .
$$

Now we have

$$
\begin{aligned}
& h^{\prime}(z)=C e^{C z+D}-e^{r z+s}\left\{r-p(r+C) e^{C z+D}\right\}, \\
& h^{\prime \prime}(z)=C^{2} e^{C z+D}-e^{r z+s}\left\{r^{2}-p(r+C)^{2} e^{C z+D}\right\}, \quad \text { and } \\
& h^{\prime \prime \prime}(z)=C^{3} e^{C z+D}-e^{r z+s}\left\{r^{3}-p(r+C)^{3} e^{C z+D}\right\} .
\end{aligned}
$$

If $p \geqq 3$, then $r=0$ and $e^{r z+s} \equiv 1$, so we have $p=2$ and $e^{r z+s}=e^{-3(C z+D)}$. Then by (3.5.0)

$$
H(z) L(z)^{2}=\frac{1}{C}\left\{e^{C z+D}-C(b+1) z-b e^{-(C z+D)}+C_{2}\right\}
$$

for a constant $C_{2}$. Substituting this into (3.5.8), we obtain a contradiction even if $b=-1$.

Next suppose that $\left(e^{C z+D}-1\right) / L(z)$ is different from zero. Then we may choose $L(z)=e^{C z+D}-1$. Hence by $(3.5 .0)$

$$
H(z)=\frac{F(z)}{L(z)^{p}}=\left(e^{C z+D}-1\right)^{-p}\left\{\int_{z_{1}}^{z} e^{-(p-1)(C \zeta+D)}\left(e^{(p-1)(C \zeta+D)}-b\right)\left(e^{C \zeta+D}-1\right) d \zeta\right\}
$$

for some point $z_{1}$ with $e^{C z_{1}+D}=1$. It follows immediately $p=2 m$, a positive integer $m$ and $b=-1$. If $m=1, H(z)=C^{-1} e^{-(C z+D)}$ and the result (10). For the case where $m>1$ it is shown that the function represented by integration always 
assumes a zero at the point where we never expect. It needs to be a zero of either $e^{p(C z+D)}+1$ or $e^{C z+D}-1$ with $I\left(z ; z_{0}\right)=0$.

Subcase ; $b=1$.

Now we choose $H$ and $L$ as

$$
F(z)=H(z) L(z)^{p+1},
$$

that is, $H(z)$ vanishes only at zeros of $e^{(p-1)(C z+D)}+\cdots+e^{C z+D}+1$. Of course, $H$ and $L$ do not have any common zero. Then we have the following equation corresponding to (3.5.1)

$$
\begin{aligned}
H^{\prime}(z) & L(z)+(p+1) H(z) L^{\prime}(z) \\
= & e^{-(p-1)(C z+D)}\left(\frac{e^{C z+D}-1}{L(z)}\right)^{p}\left(e^{(p-2)(C z+D)}+\cdots+e^{C z+D}+1\right) .
\end{aligned}
$$

After the manner of the previous case we shall investigate the behavior of the logarithmic derivative of $g$ at the zeros of $H$ and $L$. If either of them is different from zero, then let it be equal to one identically. Since (3.0.4) gives

$$
\frac{g^{\prime}(z)}{g(z)}=\frac{-1}{H(z) L(z)}\left(\frac{e^{C z+D}-1}{L(z)}\right)^{p}
$$

that is of the expansion

$$
\frac{1}{z-a} \frac{-\left(e^{C a+D}-1\right)^{p}}{H^{\prime}(a) L(a)^{p+1}}\{1+O(z-a)\}
$$

near a zero of $H, a$, and

$$
\frac{1}{z-b} \frac{-C^{p}}{H(b) L^{\prime}(b)^{p+1}}\{1+O(z-b)\}
$$

near a zero of $L, b$. The equation (3.5.9) implies

$$
\frac{-\left(e^{C a+D}-1\right)^{p}}{H^{\prime}(a) L(a)^{p+1}}=1 \text { and } \frac{-C^{p}}{H(b) L^{\prime}(b)^{p+1}}=-\frac{p+1}{p-1} .
$$

Unless $L(z) \equiv 1,(p+1) /(p-1)=1+2(p-1)^{-1}$ must be a positive integer, so that $p=2$ or $p=3$.

Firstly suppose that $L(z) \equiv 1$, then

$$
H(z)=F(z)=\int_{a}^{z} e^{-(p-1)(C \zeta+D)}\left(e^{(p-1)(C \zeta+D)}-1\right)\left(e^{C \zeta+D}-1\right)^{p-1} d \zeta .
$$

A simple analysis shows $p=2 m+1, m$ a positive integer. It is however impossible that this function vanishes only at zeros of $e^{(p-1)(C z+D)}+\cdots+e^{C z+D}+1$.

Next suppose that $L(z) \not \equiv 1$ and $p=2$. Then

$$
H(z) L(z)^{3}=\frac{1}{C}\left\{e^{C z+D}-2 C(z-b)-e^{-(C z+D)}\right\},
$$


which has infinitely many zeros. However it follows from this form that $L(z)$ can have only one zero $b$ and $H(z)$ has no zero. This is a contradiction.

Let $p$ be equal to 3 . Then we have

$$
H(z) L(z)^{4}=\frac{1}{2 C} e^{-2(C z+D)}\left(e^{C z+D}-1\right)^{4},
$$

so that it follows that $H(z) \equiv 1$ and

$$
L(z)=\omega e^{-(1 / 2)(C z+D)}\left(e^{C z+D}-1\right), \quad \omega^{4}=\frac{1}{2 C}
$$

thus $\alpha^{\prime}(z)=2 C e^{-(C z+D)}\left(e^{2(C z+D)}+e^{C z+D}+1\right)$. Hence we can write

$$
f(z)=L(z)^{-2} \exp (N(z)) \text { and } g(z)=L(z)^{-2} \exp (M(z)),
$$

by the previous observation and

$$
\begin{aligned}
& M^{\prime}(z)=\frac{g^{\prime}(z)}{g(z)}+2 \frac{L^{\prime}(z)}{L(z)}=-C\left(2 e^{C z+D}+1\right), \\
& N^{\prime}(z)=M^{\prime}(z)+\alpha^{\prime}(z)=C\left(2 e^{-(C z+D)}+1\right) .
\end{aligned}
$$

Finally we have the pair in (5), which has no influence on Corollary.

\section{$\S$ 3.6. The case where $-A / C$ is not an integer greater than -1 .}

In this case we know that $\alpha^{\prime}(z)$ does not have any zero common to $e^{C z+D}-1$. Put $\alpha^{\prime}(z)=\left(e^{A z+B}-1\right) / G(z)$ for an entire function $G$ of order at most one, which assumes the value zero possibly at the one-points of $e^{A z+B}$ with the simple multiplicity. Then (3.3.1) gives

$$
G^{\prime}(z)\left(e^{C z+D}-1\right)-A e^{C z+D} G(z)=e^{A z+B} e^{C z+D}-1 .
$$

Here if $G(z)$ is zero-free, it is denoted by $e^{r z+s}$ and then (3.6.1) leads however to a contradiction by using Lemma 2 . Hence $G$ has indeed a zero. The function $G$ is represented by a product of the following two entire functions $H$ and $L$. They are of order at most one, $H$ assumes the value zero only at onepoints of $e^{A z+B}$ but those of $e^{C z+D}$, and $L$ does at all their common one-points. Of course, all the multiplicities are simple, and there is no zero common to $H$ and $L$.

At a zero of $H, a$ say, (3.0.4) and (3.6.1) give the expansion

$$
\frac{g^{\prime}(z)}{g(z)}=\frac{1}{H^{\prime}(a) L(a)} \frac{1}{z-a}\{1+O(z-a)\}=\frac{1}{z-a}\{1+O(z-a)\} .
$$

At a zero of $L, b$ say, we have also

$$
\frac{g^{\prime}(z)}{g(z)}=\frac{1}{H(b) L^{\prime}(b)} \frac{1}{z-b}\{1+O(z-b)\}=\frac{C-A}{C+A} \frac{1}{z-b}\{1+O(z-b)\} .
$$


Hence then $(C-A) /(C+A)=(1-A / C) /(1+A / C)$ must be some integer $k$ different from $0, \pm 1,-2$, and -3 , since $-A / C=(k-1) /(k+1)$ is a rational number different from an integer $\geqq-1$. Thus $-A / C$ is not an integer.

These observations imply the pair

$$
\left\{\begin{array}{l}
f(z)=H(z) L(z)^{k} \exp (N(z)), \\
g(z)=H(z) L(z)^{k} \exp (M(z)),
\end{array}\right.
$$

for entire functions $M$ and $N$ satisfying

$$
M^{\prime} H L=1-H^{\prime} L-k H L^{\prime} \text { and } N^{\prime} H L=e^{A z+B}-H^{\prime} L-k H L^{\prime} .
$$

Calculation gives

$$
\left\{\begin{array}{l}
f^{\prime \prime}(z)=-\frac{L(z)^{k-1}\left(A+\alpha^{\prime}(z)\right)}{e^{C z+D}-1} e^{A z+B} \exp (N(z)), \\
g^{\prime \prime}(z)=-\frac{L(z)^{k-1}\left(A+\alpha^{\prime}(z)\right)}{e^{C z+D}-1} e^{C z+D} \exp (M(z)),
\end{array}\right.
$$

and, noting (3.6.3) and (3.6.1) with $G=H L$,

$$
\left\{\begin{array}{l}
f^{\prime \prime \prime}(z)=\frac{K_{1}(z) L(z)^{k-3} e^{A z+B}}{H(z)^{2}\left(e^{C z+D}-1\right)^{2}} \exp (N(z)), \\
g^{\prime \prime \prime}(z)=\frac{K_{2}(z) L(z)^{k-3} e^{C z+D}}{H(z)^{2}\left(e^{C z+D}-1\right)^{2}} \exp (M(z)),
\end{array}\right.
$$

with

$$
\begin{aligned}
K_{1}(z)= & A\left(A+C e^{C z+D}\right) H(z)^{2} L(z)^{2}+\left\{C e^{A z+B} e^{C z+B}+3 A e^{A z+B}\right. \\
& \left.-(A+C) e^{C z+D}-2 A\right\} H(z) L(z)+\left(e^{A z+B}-1\right)\left(e^{A z+B} e^{C z+D}+e^{A z+B}-2\right), \\
K_{2}(z)= & A\left(A e^{C z+D}+C\right) H(z)^{2} L(z)^{2}+\left\{2 A e^{A z+B} e^{C z+D}+(A+C) e^{A z+B}\right. \\
& \left.-3 A e^{C z+D}-C\right\} H(z) L(z)+\left(e^{A z+B}-1\right)\left(2 e^{A z+B} e^{C z+D}-e^{C z+D}-1\right) .
\end{aligned}
$$

These are valid even if $L(z)$ is zero-free by taking it as the constant 1 , for example. In the case the number $-A / C$ may be complex and $H(z)$ has indeed a zero

Supposing that $f^{\prime \prime \prime} / g^{\prime \prime \prime}=e^{\delta}$ for entire $\delta$, we have

$$
\frac{g^{\prime \prime \prime}}{g^{\prime \prime}}=\frac{\gamma^{\prime}}{e^{\delta-\gamma}-1}
$$

by the assumption (3.0.3). Since $\beta-\alpha=A z+B$ and $\beta-\gamma=C z+D$, we have

$$
\gamma=\alpha+(A z+B)-(C z+D) .
$$

Now (3.6.4) and (3.6.5) give

$$
e^{\delta}=\frac{K_{1}}{K_{2}} e^{(A z+B)-(C z+D)+\alpha} .
$$




$$
\frac{g^{\prime \prime \prime}}{g^{\prime \prime}}=-\frac{K_{2}}{H^{2} L^{2}\left(A+\alpha^{\prime}\right)\left(e^{C z+D}-1\right)},
$$

therefore

$$
\frac{\left(\alpha^{\prime}+A-C\right)}{K_{1} / K_{2}-1}=-\frac{K_{2}}{H^{2} L^{2}\left(A+\alpha^{\prime}\right)\left(e^{C z+D}-1\right)} .
$$

Hence, noting $H L=G=\left(e^{A z+B}-1\right) / \alpha^{\prime}$, we put $K_{1} / K_{2}=e^{r z+s} \not \equiv 1$ and we obtain

$$
\begin{aligned}
\left(e^{r z+s}-1\right)\left[\left(2 e^{A z+B} e^{C z+D}-e^{C z+D}-1\right) \alpha^{\prime 2}+\left\{2 A e^{A z+B} e^{C z+D}+(A+C) e^{A z+B}\right.\right. \\
\\
\left.\left.-3 A e^{C z+D}-C\right\} \alpha^{\prime}+A\left(A e^{C z+D}+C\right)\left(e^{A z+B}-1\right)\right] \\
=-\left(e^{A z+B}-1\right)\left(e^{C z+D}-1\right)\left(\alpha^{\prime}+A\right)\left(\alpha^{\prime}+A-C\right) .
\end{aligned}
$$

Then we have the following quadratic equation in $\alpha^{\prime}$,

$$
a_{2}(z) \alpha^{\prime}(z)^{2}+a_{1}(z) \alpha^{\prime}(z)+a_{0}(z)=0,
$$

with

$$
\begin{aligned}
a_{2}(z)= & \left(e^{r z+s}-1\right)\left(2 e^{A z+B} e^{C z+D}-e^{C z+D}-1\right)+\left(e^{A z+B}-1\right)\left(e^{C z+D}-1\right), \\
a_{1}(z)= & \left(e^{r z+s}-1\right)\left(2 A e^{A z+B} e^{C z+D}+(A+C) e^{A z+B}-3 A e^{C z+D}-C\right) \\
& +(2 A-C)\left(e^{A z+B}-1\right)\left(e^{C z+D}-1\right)
\end{aligned}
$$

and

$$
a_{0}(z)=A\left\{\left(e^{r z+s}-1\right)\left(A e^{C z+D}+C\right)\left(e^{A z+B}-1\right)+(A-C)\left(e^{A z+B}-1\right)\left(e^{C z+D}-1\right)\right\} .
$$

If the coefficient $a_{2}$ vanishes identically, Lemma 2 shows that the only possibility is that $e^{C z+D}=e^{-2(A z+B)}$ and $e^{r z+s}=-e^{A z+B}$. Then the equation (3.6.6) has no desired solution $\alpha^{\prime}$. Consequently the function $a_{1}{ }^{2}-4 a_{2} a_{0}$ needs to be the square of a meromorphic function. Since it is equal to

$$
C^{2}\left(e^{A z+B}-1\right)^{2}\left(e^{C z+D}-1\right)^{2}-2 b_{1}(z)\left(e^{A z+B}-1\right)\left(e^{C z+D}-1\right)\left(e^{r z+s}-1\right)+b_{0}(z)\left(e^{r z+s}-1\right)^{2},
$$

with

$$
\begin{aligned}
b_{1}(z)= & 2 A(A-C) e^{A z+B} e^{C z+D}-(2 A+C)(A-C) e^{A z+B} \\
& +A(2 A-C) e^{C z+D}-\left(2 A^{2}-2 A C+C^{2}\right), \\
b_{0}(z)= & -4 A^{2} e^{2(A z+B)} e^{2(C z+D)}+4 A(A-C) e^{2(A z+B)} e^{C z+D}+(A+C)^{2} e^{2(A z+B)} \\
& -2 A(A-C) e^{A z+B} e^{C z+D}+2 C(A-C) e^{A z+B}+5 A^{2} e^{2(C z+D)} \\
& -2 A(2 A-C) e^{C z+D}+C(C-4 A),
\end{aligned}
$$

to do this we require that $b_{1}(z)^{2}-C^{2} b_{0}(z)$ should vanish identically. Then 


$$
\begin{aligned}
\frac{1}{4 A^{2}}\left\{b_{1}(z)^{2}-C^{2} b_{0}(z)\right\}= & C_{1} e^{2(A z+B)} e^{2(C z+D)}-C_{2} e^{2(A z+B)} e^{C z+D}+C_{3} e^{2(A z+B)} \\
& +C_{2} e^{A z+B} e^{2(C z+D)}-C_{2} e^{A z+B} e^{C z+D}+C_{2} e^{A z+B}+C_{3} e^{2(C z+D)} \\
& -C_{2} e^{C z+D}+C_{1},
\end{aligned}
$$

with $C_{1}=A^{2}-2 A C+2 C^{2}, C_{2}=(A-C)(2 A-C)$ and $C_{3}=A^{2}-A C-C^{2}$. It follows from Lemma 2 that Borel's identity $b_{1}(z)^{2}-C^{2} b_{0}(z)=0$ is impossible in the present situation.

This completes our proof.

\section{REFERENCES}

[1] K.F. Barth, D. A. Brannan and W. K. Hayman, Research problems in complex analysis, Bull. London Math. Soc., 16 (1984), 490-517.

[2] F. Gross, Factorization of meromorphic functions, U.S. Gov. Printing Office, 1972.

[3] G. G. Gundersen, When two entire functions and also their first derivatives have the same zeros, Indiana University Math. J., Vol. 30, No. 2 (1981), 293-303.

[4] W.K. Hayman, Meromorphic functions, Clarendon Press, Oxford 1964.

[5] E. Hille, Ordinary differential equations in the complex domain, Wiley \& Sons, New York (1976).

[6] G. Hiromi, and M. Ozawa, On the existence of analytic mappings between two ultrahyperelliptic surfaces, Kōdai Math. Sem. Rep., 17 (1965), 281-306.

[7] C.F. OsGood AND C.C. YANG, On the quotient of two integral functions, J. Math. Anal. Appl. 54 (1976), 408-418.

Department of Mathematics

SCIENCE University of TOKYO

NODA, CHIBA, JAPAN 